\title{
Refinements for mean-inequalities via the stabilizability concept
}

Mustapha Raïssouli

Correspondence:

raissouli_10@hotmail.com Department of Mathematics,

Faculty of Science, Taibah University, Al Madinah Al

Munawwarah, P.O. Box 30097, Zip Code 41477, Kingdom of Saudi Arabia

\begin{abstract}
Exploring the stabilizability concept, recently introduced by Raïssouli, we give an approach for obtaining refinements of mean-inequalities in a general point of view. Our theoretical study will be illustrated by a lot of examples showing the generality of our approach and the interest of the stabilizability concept.
\end{abstract}

AMS Subject Classification: 26E60.

Keywords: means, refinements of mean-inequalities, stable and stabilizable means

\section{Introduction}

Stability and stabilizability concepts for binary means have been recently introduced by Raïssouli [1]. The aim of this article is to show that the above concepts are useful tool from the theoretical point of view as well as for practical purposes. Let us first recall some basic notions about binary means that will be needed throughout the article. We understand by mean a binary map $m$ between positive real numbers satisfying the following statements.

(i) $m(a, a)=a$, for all $a>0$;

(ii) $m(a, b)=m(b, a)$, for all $a, b>0$;

(iii) $m(t a, t b)=t m(a, b)$, for all $a, b, t>0$;

(iv) $m(a, b)$ is an increasing function in $a$ (and in $b$ );

(v) $m(a, b)$ is a continuous function of $a$ and $b$.

The set of all means can be equipped with a partial ordering, called point-wise order, defined by, $m_{1} \leq m_{2}$ if and only if $m_{1}(a, b) \leq m_{2}(a, b)$ for every $a, b>0$. We write $m_{1}$ $<m_{2}$ if and only if $m_{1}(a, b)<m_{2}(a, b)$ for all $a, b>0$ with $a \neq b$. Clearly, $m_{1}<m_{2}$ implies $m_{1} \leq m_{2}$.

The standard examples of means satisfying the above requirements are recalled in the following.

$$
\begin{aligned}
A & :=A(a, b)=\frac{a+b}{2} ; G:=G(a, b)=\sqrt{a b} ; H:=H(a, b)=\frac{2 a b}{a+b} ; \\
L & :=L(a, b)=\frac{b-a}{\ln b-\ln a}, L(a, a)=a ; I:=I(a, b)=\frac{1}{e}\left(\frac{b^{b}}{a^{a}}\right)^{1 /(b-a)}, I(a, a)=a,
\end{aligned}
$$

respectively called the arithmetic, geometric, harmonic, logarithmic, and identric means. These means satisfy the following inequalities

(C) 2012 Raïssouli; licensee Springer. This is an Open Access article distributed under the terms of the Creative Commons Attribution License (http://creativecommons.org/licenses/by/2.0), which permits unrestricted use, distribution, and reproduction in any medium, provided the original work is properly cited. 


$$
\min <H<G<L<I<A<\max
$$

where min and max are the trivial means $(a, b) \mapsto \min (a, b)$ and $(a, b) \mapsto \max (a, b)$. For a given mean $m$, we set

$$
m^{*}(a, b)=\left(m\left(a^{-1}, b^{-1}\right)\right)^{-1},
$$

and it is easy to see that $m^{*}$ is also a mean, called the dual mean of $m$. The symmetry and homogeneity axioms (ii), (iii) yield

$$
m^{*}(a, b)=\frac{a b}{m(a, b)}
$$

for all $a, b>0$, which we briefly write $m^{*}=G^{2} / m$. Every mean $m$ satisfies $m^{* *}=m$ and, if $m_{1}$ and $m_{2}$ are two means such that $m_{1} \leq m_{2}$ (resp. $\left.m_{1}<m_{2}\right)$ then $m_{1}^{*} \geq m_{2}^{*}$ (resp. $m_{1}^{*}>m_{2}^{*}$ ). It is clear that the arithmetic and harmonic means are mutually dual and the geometric mean is the unique self-dual mean. We recall that, the mean-map $m \mapsto m^{*}$ is point-wise convex in the sense that the following inequality [1]

$$
\left((1-t) m_{1}+t m_{2}\right)^{*} \leq(1-t) m_{1}^{*}+t m_{2}^{*}
$$

holds true for every real number $t \in[0,1]$ and all means $m_{1}$ and $m_{2}$. Further, the inequality (1.5) is strict (in the above sense) if and only if $t \in(0,1)$ and $m_{1} \neq m_{2}$.

The dual of the logarithmic mean is given by

$$
L^{*}:=L^{*}(a, b)=a b \frac{\ln b-\ln a}{b-a}, L^{*}(a, a)=a,
$$

while that of the identric mean is

$$
I^{*}:=I^{*}(a, b)=e\left(\frac{a^{b}}{b^{a}}\right)^{1 / b-a}, I^{*}(a, a)=a .
$$

The following inequalities are immediate from the above.

$$
\min <H<I^{*}<L^{*}<G<L<I<A<\max .
$$

A mean $m$ is called strict if $m(a, b)$ is strictly monotonic increasing in $a$ (and in $b$ ). Every strict mean $m$ satisfies that, $m(a, b)=a \Rightarrow a=b$. It is easy to see that if $m$ is a strict mean then so is $m^{*}$. The means min and max are not strict while $H, G, A, L, L^{*}$, $I, I^{*}$ are strict means.

In the literature, there are some families of means, called power means, which include the above familiar means. Precisely, let $p$ be a real number, we recall the following:

- The power binomial mean:

$$
\left\{\begin{array}{l}
B_{p}(a, b):=B_{p}=\left(\frac{a^{p}+b^{p}}{2}\right)^{1 / p}, \\
B_{-1}=H, B_{1}=A, B_{0}:=\lim _{p \rightarrow 0} B_{p}=G .
\end{array}\right.
$$


- The power logarithmic mean:

$$
\left\{\begin{array}{l}
L_{p}(a, b)=L_{p}=\left(\frac{a^{p+1}-b^{p+1}}{(p+1)(a-b)}\right)^{1 / p}, L_{p}(a, a)=a, \\
L_{-2}=G, L_{-1}=L, L_{0}=I, L_{1}=A .
\end{array}\right.
$$

- The power difference mean:

$$
\left\{\begin{array}{l}
D_{p}(a, b):=D_{p}=\frac{p}{p+1} \frac{a^{p+1}-b^{p+1}}{a^{p}-b^{p}}, D_{p}(a, a)=a, \\
D_{-2}=H, D_{-1}=L^{*}, D_{-1 / 2}=G, D_{0}=L, D_{1}=A .
\end{array}\right.
$$

- The power exponential mean:

$$
\left\{\begin{array}{l}
I_{p}(a, b):=I_{p}=\exp \left(-\frac{1}{p}+\frac{a^{p} \ln a-b^{p} \ln b}{a^{p}-b^{p}}\right), I_{p}(a, a)=a, \\
I_{-1}=I^{*}, I_{0}=G, I_{1}=I .
\end{array}\right.
$$

- The second power logarithmic mean:

$$
\left\{\begin{array}{l}
l_{p}(a, b):=l_{p}=\left(\frac{1}{p} \frac{b^{p}-a^{p}}{\ln b-\ln a}\right)^{1 / p}, l_{p}(a, a)=a, \\
l_{-1}=L^{*}, l_{0}=G, l_{1}=L .
\end{array}\right.
$$

If $m_{p}$ stands for one of the above power means, it is well known that $m_{-\infty}=\min$ and $m_{+\infty}=$ max. Further, all the above power means (also called means of order $p$ ) are strictly monotonic increasing in $p$, for fixed $a, b>0$. Otherwise, it is easy to see that $B_{p}^{*}=B_{-p}$ for all real number $p$. We notice that these power means are included in a generalized family of means (not needed here), namely the Stolarsky mean of order 2, see [2] for instance.

In the past years, enormous efforts by some authors has been devoted to refine various inequalities between means (called mean-inequalities), see [2-10] for instance and the related references cited therein. Our fundamental goal in this article is to explore the stabilizability concept for obtaining a game of mean-inequalities whose certain of them have been differently discussed in the literature. Our approach stems its importance in the following items:

First, by a united procedure we find some known mean-inequalities and further other ones in a short and nice manner.

Second, by the same procedure, starting from an arbitrary lower and/or upper bounds of a stabilizable mean we show how to obtain in a recursive manner an infinity of lower and/or upper bounds of this mean. We also give, throughout a lot of examples, sufficient conditions for ensuring that the new bounds are refinements of the initial ones. 


\section{Background material about stabilizable means}

For the sake of simplicity for the reader, we will recall in this section some basic notions and results stated by Raïssouli in an earlier article [1].

Definition 2.1. Let $m_{1}, m_{2}$, and $m_{3}$ be three given means. For all $a, b>0$, define

$$
\mathcal{R}\left(m_{1}, m_{2}, m_{3}\right)(a, b)=m_{1}\left(m_{2}\left(a, m_{3}(a, b)\right), m_{2}\left(m_{3}(a, b), b\right)\right) ，
$$

called the resultant mean-map of $m_{1}, m_{2}$ and $m_{3}$.

A study investigating the elementary properties of the resultant mean-map has been stated in [1]. Here, we just recall the following result needed later.

Proposition 2.1. ([1], Proposition 1) The map $(a, b) \mapsto R\left(m_{1}, m_{2}, m_{3}\right)(a, b)$ defines a mean, with the following properties:

(i) For every means $m_{1}, m_{2}, m_{3}$ we have

$$
\left(\mathcal{R}\left(m_{1}, m_{2}, m_{3}\right)\right)^{*}=\mathcal{R}\left(m_{1}^{*}, m_{2}^{*}, m_{3}^{*}\right) .
$$

(ii) The mean-map $\mathcal{R}$ is point-wisely increasing with respect to each its mean variables, that is,

$$
\left(m_{1} \leq m_{1}^{\prime}, m_{2} \leq m_{2}^{\prime}, m_{3} \leq m_{3^{\prime}}^{\prime}\right) \Rightarrow \mathcal{R}\left(m_{1}, m_{2}, m_{3}\right) \leq \mathcal{R}\left(m_{1}^{\prime}, m_{2}^{\prime}, m_{3}^{\prime}\right) .
$$

The following result, which the proof is straightforward, is also of interest in what follows.

Proposition 2.2. ([1], Proposition 2) For all mean $M$, the mean-map $m \mapsto \mathcal{R}(A, m, M)$ is point-wise affine in the sense that the mean-equality

$$
\mathcal{R}\left(A,(1-t) m+t m^{\prime}, M\right)=(1-t) \mathcal{R}(A, m, M)+t \mathcal{R}\left(A, m^{\prime}, M\right)
$$

holds for all real number $t \in[0,1]$ and all means $m, m^{\prime}$.

Example 2.1. Simple computations lead to

$$
\begin{aligned}
& \mathcal{R}(H, H, A)=\left(\frac{1}{2} A+\frac{1}{2} H\right)^{*}=\frac{2 A H}{A+H}, \mathcal{R}(H, A, A)=\frac{3}{4} A+\frac{1}{4} H . \\
& \mathcal{R}(A, G, G)=\left(\frac{1}{2} A G+\frac{1}{2} G^{2}\right)^{1 / 2}, \mathcal{R}(A, A, G)=\frac{1}{2} A+\frac{1}{2} G . \\
& \mathcal{R}(G, G, A)=\sqrt{A G}, \mathcal{R}(G, A, A)=\left(\frac{3}{4} A^{2}+\frac{1}{4} G^{2}\right)^{1 / 2} .
\end{aligned}
$$

The following lemma will be needed in the sequel.

Lemma 2.3. ([1], Example 5) Let $m_{1}$ and $m_{2}$ be two means, then the following equality

$$
\mathcal{R}\left(m_{1}, m_{2}, G\right)(a, b)=m_{1}(\sqrt{a}, \sqrt{b}) m_{2}(\sqrt{a}, \sqrt{b}) .
$$

holds for all $a, b>0$.

As proved in [1], and will be again shown throughout this article, the resultant mean-map stems its importance in the fact that it is a tool for introducing the stability and stabilizability notions as recalled in the following.

Definition 2.2. A mean $m$ is said to be: 
(a) Stable if $\mathcal{R}(m, m, m)=m$.

(b) Stabilizable if there exist two nontrivial stable means $m_{1}$ and $m_{2}$ satisfying the relation $\mathcal{R}\left(m_{1}, m, m_{2}\right)=m$. We then say that $m$ is $\left(m_{1}, m_{2}\right)$-stabilizable.

In [1], Raïssouli stated a developed study about the stability and stabilizability of the standard and power means. In particular, he proved that if $m$ is stable then so is $m^{*}$, and if $m$ is $\left(m_{1}, m_{2}\right)$-stabilizable then $m^{*}$ is $\left(m_{1}^{*}, m_{2}^{*}\right)$-stabilizable. About the power standard means, the summarized results stated in [1] are recited in the following theorem.

Theorem 2.4. ([1], Theorems 1,3,4,5) For all real number $p$, the following statements are met:

(1) The power binomial mean $B_{p}$ is stable.

(2) The power logarithmic mean $L_{p}$ is $\left(B_{p}, A\right)$-stabilizable, while the power difference mean $D_{p}$ is $\left(A, B_{p}\right)$-stabilizable.

(3) The power exponential mean $I_{p}$ is $\left(G, B_{p}\right)$-stabilizable, while the second power logarithmic mean $l_{p}$ is $\left(B_{p}, G\right)$-stabilizable.

The following result, needed in the sequel, is immediate from the above.

Corollary 2.5. With the above, the following assertions are met:

(1) The arithmetic, geometric, and harmonic means $A, G$ and $H$ are stable.

(2) The logarithmic mean $L$ is $(H, A)$-stabilizable and $(A, G)$-stabilizable while the identric mean $I$ is $(G, A)$-stabilizable.

(3) The mean $L^{*}$ is $(A, H)$-stabilizable and $(H, G)$-stabilizable while $I^{*}$ is $(G, H)$ stabilizable.

N.B. Throughout the article, we investigate some results of mean-inequalities, under convenient assumptions, for the strict symbol < (in the above sense). By similar manner, all stated results remain still true when we replace $<$ by $\leq$ in the hypotheses as in the related conclusions. Of course, this is not immediate since $m_{1}<m_{2}$ is, as hypothesis and as conclusion, stronger than $m_{1} \leq m_{2}$.

\section{Refinements for mean-inequalities: general approach}

As already pointed before, this section displays some important applications of the above concepts for refining mean-inequalities in a general point of view. Particular examples illustrating the generality of our approach and the interest of this study will be discussed. We first state the following result which is an improvement of that of Proposition 2.1.

Theorem 3.1. Let $m_{1}, m_{1}^{\prime}, m_{2}, m_{2}^{\prime}, m_{3}$, and $m_{3}^{\prime}$ be means such that

$$
m_{1} \leq m_{1}^{\prime}, m_{2} \leq m_{2}^{\prime} \text { and } m_{3} \leq m_{3}^{\prime} .
$$

Assume that one of the following three statements holds:

(i) $m_{1}<m_{1}^{\prime}, m_{2}^{\prime}$ and $m_{3}^{\prime}$ are strict means,

(ii) $m_{2}<m_{2}^{\prime}, m_{1}$ and $m_{3}^{\prime}$ are strict means,

(iii) $m_{3}<m_{3}^{\prime}, m_{1}$ and $m_{2}$ are strict means.

Then we have

$$
\mathcal{R}\left(m_{1}, m_{2}, m_{3}\right)<\mathcal{R}\left(m_{1}^{\prime}, m_{2}^{\prime}, m_{3}^{\prime}\right),
$$

in the sense that

$$
\mathcal{R}\left(m_{1}, m_{2}, m_{3}\right)(a, b)<\mathcal{R}\left(m_{1}^{\prime}, m_{2}^{\prime}, m_{3}^{\prime}\right)(a, b)
$$


holds for all $a, b>0$ with $a \neq b$.

Proof. Assume that (3.1) holds:

(i) Without loss the generality, let $a, b>0$ with $a<b$. Then we have

$$
\begin{aligned}
\mathcal{R}\left(m_{1}, m_{2}, m_{3}\right)(a, b) & =m_{1}\left(m_{2}\left(a, m_{3}(a, b)\right), m_{2}\left(m_{3}(a, b), b\right)\right) \\
& \leq m_{1}\left(m_{2}^{\prime}\left(a, m_{3}^{\prime}(a, b)\right), m_{2}^{\prime}\left(m_{3}^{\prime}(a, b), b\right)\right) .
\end{aligned}
$$

Since $m_{3}^{\prime}$ and $m_{2}^{\prime}$ are assumed strict means then we have, respectively,

$$
a<m_{3}^{\prime}(a, b)<b \text { and } m_{2}^{\prime}\left(a, m_{3}^{\prime}(a, b)\right)<m_{2}^{\prime}\left(m_{3}^{\prime}(a, b), b\right) .
$$

This, with $m_{1}<m_{1}^{\prime}$, yields the desired result.

(ii), (iii) Similar to (i). We left the detail to the reader as simple exercise.

Now, we are in position to state the following result which gives a refinement of a mean-inequality $m_{1}<m<m_{2}$ when $m$ is $\left(m_{1}, m_{2}\right)$-stabilizable or $\left(m_{2}, m_{1}\right)$-stabilizable.

Theorem 3.2. Let $m$ be a $\left(m_{1}, m_{2}\right)$-stabilizable mean with $m_{1}$ and $m_{2}$ are strict means. Assume that $m_{1}<m<m_{2}$, then the following refinement holds

$$
m_{1}<\mathcal{R}\left(m_{1}, m_{1}, m_{2}\right)<m<\mathcal{R}\left(m_{1}, m_{2}, m_{2}\right)<m_{2} .
$$

If $m_{2}<m<m_{1}$ then the role of $m_{1}$ and $m_{2}$ in the above inequalities is reversed.

Proof. According to Theorem 3.1, with $m_{1}<m<m_{2}$ and the fact that $m_{1}$ and $m_{2}$ are strict means, we obtain

$$
\begin{aligned}
\mathcal{R}\left(m_{1}, m_{1}, m_{1}\right) & <\mathcal{R}\left(m_{1}, m_{1}, m_{2}\right)<\mathcal{R}\left(m_{1}, m_{1} m_{2}\right) \\
& <\mathcal{R}\left(m_{1}, m_{2}, m_{2}\right)<\mathcal{R}\left(m_{2}, m_{2}, m_{2}\right) .
\end{aligned}
$$

This, with the fact that $m_{1}$ and $m_{2}$ are stable and $m$ is $\left(m_{1}, m_{2}\right)$-stabilizable, yields the desired result.

Now, let us observe the following particular examples illustrating the situation of the above theorem.

Example 3.1. Knowing that $H<L<A$ with $L$ is $(H, A)$-stabilizable, the above theorem gives

$$
H<\mathcal{R}(H, H, A)<L<\mathcal{R}(H, A, A)<A .
$$

This, with (2.5), gives the following refinement of the arithmetic-logarithmic-harmonic mean inequality

$$
H<\frac{2 G^{2}}{A+H}<L<\frac{3}{4} A+\frac{1}{4} H<A .
$$

Example 3.2. Starting from $G<L<A$ with $L$ is $(A, G)$-stabilizable, Theorem 3.2 implies that

$$
G<\mathcal{R}(A, G, G)<L<\mathcal{R}(A, A, G)<A .
$$

According to (2.6), we obtain the following inequalities which refine the arithmeticlogarithmic-geometric mean inequality

$$
G<\left(\frac{1}{2} A G+\frac{1}{2} G^{2}\right)^{1 / 2}<L<\frac{1}{2} A+\frac{1}{2} G<A .
$$


Example 3.3. Now, consider the known inequalities $G<I<A$ with $I$ is $(G, A)$ stabilizable.

Similarly to the above we obtain

$$
G<\mathcal{R}(G, G, A)<I<\mathcal{R}(G, A, A)<A .
$$

This, when combined with (2.7), implies a refinement of the arithmetic-identric-geometric mean inequality given by

$$
G<\sqrt{A G}<I<\left(\frac{3}{4} A^{2}+\frac{1}{4} G^{2}\right)^{1 / 2}<A .
$$

Refinements of mean-inequalities, even stronger than that of the above examples, are largely studied in the literature, see [2] and the related reference cited therein. As already pointed before, our approach gives a united procedure having a general point of view when we have to refine a mean double inequality $m_{1} \leq m \leq m_{2}$ where the intermediary mean $m$ is $\left(m_{1}, m_{2}\right)$-stabilizable or $\left(m_{2}, m_{1}\right)$-stabilizable. Further, the next theorem shows that our approach can be successively repeated in the aim to obtain more lower and/or upper bounds of a given stabilizable mean.

Theorem 3.3. Let $m$ be a $\left(m_{1}, m_{2}\right)$-stabilizable mean with $m_{1}$ and $m_{2}$ are strict means. Let

$m_{3}$ and $m_{4}$ be two means such that

$$
m_{3}<m<m_{4} .
$$

Then we have the following mean-inequalities

$$
\mathcal{R}\left(m_{1}, m_{3}, m_{2}\right)<m<\mathcal{R}\left(m_{1}, m_{4}, m_{2}\right) .
$$

Proof. By Theorem 3.1, with $m_{3}<m<m_{4}$, we have

$$
\mathcal{R}\left(m_{1}, m_{3}, m_{2}\right)<\mathcal{R}\left(m_{1}, m, m_{2}\right)<\mathcal{R}\left(m_{1}, m_{4}, m_{2}\right) .
$$

This, with the fact that $m$ is $\left(m_{1}, m_{2}\right)$-stabilizable, gives the desired result.

As pointed in the above, Theorem 3.3 starts from an arbitrary lower and upper bounds of a stabilizable mean $m$ for giving other lower and upper bounds of the mean $m$, and so we can iterate the same procedure for obtaining an infinity of lower and upper bounds of $m$. An important question arises from this latter situation: Under what general conditions, (3.16) is a refinement of (3.15), that is,

$$
m_{3}<\mathcal{R}\left(m_{1}, m_{3}, m_{2}\right) \text { and } \mathcal{R}\left(m_{1}, m_{4}, m_{2}\right)<m_{4} \text { ? }
$$

This makes appear in (3.17) weak conditions of stabilizability, which we call sub-stabilizability and super-stabilizability of $m_{3}$ and $m_{4}$, see [11]. For the moment, we will not give any answer about general sufficient conditions for ensuring the above refinement, but we just discuss (in the sections below) the response for some particular cases.

N.B. Let $m_{p} \in\left\{l_{p}, L_{p}, I_{p}, D_{p}\right\}$ be a power mean. Henceforth, when we say

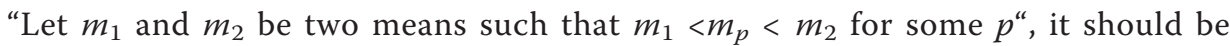
understood in the following sense,

"Let $p$ be a real number and assume that there exist two means $m_{1}:=m_{1}(p)$ and $m_{2}$ : $=m_{2}(p)$ satisfying that $m_{1}<m_{p}<m_{2}{ }^{\prime \prime}$. 


\section{Refinements for bounding the means $I_{p}$ and $L$}

Since $l_{p}$ is $\left(B_{p}, G\right)$-stabilizable, we then will be interested by bounds of $l_{p}$ in terms of $B_{p}$ and $G$.

It is worth noticing that, for given $p$, bounds of $l p$ in the form $B_{p}^{\alpha} G^{1-\alpha}$ (resp., $\alpha B_{p}+$ $(1-\alpha) \mathrm{G})$ exist for some $\alpha \in[0,1]$. This follows from (1.2) with the relationships

$$
L\left(a^{2}, b^{2}\right)=L(a, b) A(a, b),\left(l_{p}(a, b)\right)^{p}=L\left(a^{p}, b^{p}\right), ;\left(B_{p}(a, b)\right)^{p}=A\left(a^{p}, b^{p}\right)
$$

valid for all $a, b>0$ and $p \neq 0$.

We begin by regarding bounds of $l_{p}$ in a convex-geometric form $B_{p}^{\alpha} G^{1-\alpha}$ as well:

Theorem 4.1. Let $\alpha, \beta \in[0,1]$ be such that

$$
B_{p}^{\alpha} G^{1-\alpha}<l_{p}<B_{p}^{\beta} G^{1-\beta}
$$

for some $p$. Then there holds

$$
G^{\frac{1-\alpha}{2}} B_{p / 2}^{\frac{1+\alpha}{2}}=G^{\frac{1-\alpha}{2}}\left(\frac{B_{p}^{p}+G^{p}}{2}\right)^{\frac{1+\alpha}{2}}<l_{p}<G^{\frac{1-\beta}{2}}\left(\frac{B_{p}^{p}+G^{p}}{2}\right)^{\frac{1+\beta}{2 p}}=G^{\frac{1-\beta}{2}} B_{p / 2}^{\frac{1+\beta}{2}} .
$$

Proof. Since $l p$ is $(B p, G)$-stabilizable then Theorem 3.2 gives

$$
\mathcal{R}\left(B_{p}, B_{p}^{\alpha} G^{1-\alpha}, G\right)<l_{p}<\mathcal{R}\left(B_{p}, B_{p}^{\beta} G^{1-\beta}, G\right) .
$$

According to Lemma 2.3 we have, for all $a, b>0$,

$$
\begin{aligned}
\mathcal{R}\left(B_{p}, B_{p}^{\alpha} G^{1-\alpha}, G\right)(a, b) & =B_{p}(\sqrt{a}, \sqrt{b})\left(B_{p}^{\alpha} G^{1-\alpha}\right)(\sqrt{a}, \sqrt{b}) \\
& =B_{p}^{1+\alpha}(\sqrt{a}, \sqrt{b}) G^{1-\alpha}(\sqrt{a}, \sqrt{b}) .
\end{aligned}
$$

For all $a, b>0$, we can write $G(\sqrt{a}, \sqrt{b})=G^{1 / 2}(a, b)$ and it is easy to verify that,

$$
B_{p}(\sqrt{a}, \sqrt{b})=\left(\frac{B_{p}^{p}+G^{p}}{2}\right)^{1 / 2 p}(a, b)=B_{p / 2}^{1 / 2}(a, b)
$$

from which the desired double inequality (4.3) follows.

Corollary 4.2. Let $\alpha, \beta \in[0,1]$ be two real numbers such that

$$
A^{\alpha} G^{1-\alpha}<L<A^{\beta} G^{1-\beta} .
$$

Then there holds

$$
G^{\frac{1-\alpha}{2}}\left(\frac{A+G}{2}\right)^{\frac{1+\alpha}{2}}<L<G^{\frac{1-\beta}{2}}\left(\frac{A+G}{2}\right)^{\frac{1+\beta}{2}} .
$$

Proof. Taking $p=1$ in the above theorem, with the fact that $l_{1}=L$ and $B_{1}=A$, we immediately obtain the announced result.

Let us now examine the following examples in the aim to illustrate the above theoretical results.

Example 4.1. It is not hard to verify that $G<l_{p}<B_{p}$ for every $p>0$, with reversed double inequality for $p<0$. Theorem 4.1 immediately gives (with $\alpha=0, \beta=1$ for $p>$ $0 ; \alpha=1, \beta=0$ for $p<0$ ) 


$$
G^{1 / 2}\left(\frac{B_{p}^{p}+G^{p}}{2}\right)^{1 / 2 p}<l_{p}<\left(\frac{B_{p}^{p}+G^{p}}{2}\right)^{1 / p}
$$

for each real number $p \neq 0$. It is easy to verify that the double inequality (4.9) refines the initial one. In particular, we have

$$
G<\left(\frac{A G+G^{2}}{2}\right)^{1 / 2}<L<\frac{A+G}{2}<A,
$$

which refines the arithmetic-logarithmic-geometric mean inequality $G<L<A$.

Theorem 4.3. Let $\alpha \in[0,1]$ be such that

$$
B_{p}^{\alpha} G^{1-\alpha}<(>) l_{p}
$$

for some $p>(<) 0$, respectively. Then one has

$$
B_{p}^{\frac{1+\alpha}{4}} G^{\frac{3-\alpha}{4}}<(>) l_{p}
$$

If moreover $\alpha<(>) 1 / 3$ then (4.12) refines (4.11).

Proof. Assume that

$$
B_{p}^{\alpha} G^{1-\alpha}<l_{p}
$$

for some $p>0$. According to Theorem 4.1, the first inequality of (4.3) holds and the arithmetic-geometric mean inequality gives

$$
\left(\frac{B_{p}^{p}+G^{p}}{2}\right)^{\frac{1+\alpha}{2 p}}>B_{p}^{\frac{1+\alpha}{4}} G^{\frac{1+\alpha}{4}} .
$$

The desired inequality follows after a simple reduction. Further, the inequality

$$
B_{p}^{\alpha} G^{1-\alpha}<B_{p}^{\frac{1+\alpha}{4}} G^{\frac{3-\alpha}{4}}
$$

for $p>0$ is reduced to

$$
G^{\frac{1-3 \alpha}{4}}<B_{p}^{\frac{1-3 \alpha}{4}}
$$

which holds when $\alpha<1 / 3$. For the reversed inequalities, the same arguments as previous study, so completes the proof.

If we get $p=1$ in the above theorem, we immediately obtain the following result.

Corollary 4.4. Let $\alpha \mathrm{L}[0,1]$ be a real number satisfying that

$$
A^{\alpha} G^{1-\alpha}<L .
$$

Then one has

$$
A^{\frac{1+\alpha}{4}} G^{\frac{3-\alpha}{4}}<L .
$$

If moreover $\alpha<1 / 3$ then (4.18) refines (4.17).

Theorem 4.3 tells us that every given bound of $l_{p}$ in a convex-geometric form yields another bound of $l_{p}$ in an analogs, but different, form. Illustrating this latter point, we 
will deduce a better bound of $l_{p}$ than the above ones. Precisely, we may state the next result.

Theorem 4.5. Let $p$ be a real number. If $p>0$ then one has

$$
B_{p}^{1 / 3} G^{2 / 3}<l_{p} .
$$

If $p<0$ then the above inequality is reversed. In particular the following inequality holds true

$$
A^{1 / 3} G^{2 / 3}<L .
$$

Proof. Assume that $p>0$. Starting from $G<l_{p}$ (see Example 4.1), we are in the situation of Theorem 4.3 with $\alpha=0$, and so we have $B_{p}^{1 / 4} G^{3 / 4}<l_{p}$. Let us iterate successively this procedure: if in the step $n$, we have

$$
B_{p}^{\alpha_{n}} G^{1-\alpha_{n}}<l_{p}
$$

then in the step $n+1$, we obtain

$$
B_{p}^{\frac{1+\alpha_{n}}{4}} G^{\frac{3-\alpha_{n}}{4}}<l_{p}
$$

that is,

$$
B_{p}^{\alpha_{n+1}} G^{1-\alpha_{n+1}}<l_{p} \text { with } \alpha_{n+1}=\frac{1+\alpha_{n}}{4}, \alpha_{0}=\alpha .
$$

It is easy to see that the real sequences $\left(\alpha_{n}\right)_{n}$ converges to $1 / 3$ for every given initial data $\alpha_{0} \in[0,1]$. The desired inequality follows by letting $n \rightarrow+\infty$ in the recursive inequality

$$
B_{p}^{\alpha_{n}} G^{1-\alpha_{n}}<l_{p} .
$$

The proof is similar for $p<0$. Taking $p=1$ in (4.19) we obtain (4.20), so completes the proof.

To understand the interest of the above theorem, let us observe the following example.

Example 4.2. Let us apply Theorem 4.3 to the previous inequality $B_{p}^{1 / 3} G^{2 / 3}<(>) l_{p}$. Then, the next inequality

$$
l_{p}^{3 p}>G^{p}\left(\frac{B_{p}^{p}+G^{p}}{2}\right)^{2}
$$

holds true for each real number $p(p \neq 0)$. In particular, taking $p=1$ we obtain

$$
G\left(\frac{A+G}{2}\right)^{2}<L^{3}
$$

which refines $A^{1 / 3} G^{2 / 3}<L$.

Remark 4.1. The inequality (4.20) was proved by Leach and Sholander [6], while (4.26) has been shown by Sāndor [10]. These two inequalities were proved by different methods therein while together obtained here via the same approach. In the same sense, other examples will be seen later (see Remarks 4.4, 4.5, and 5.1). 
Remark 4.2. As well known, inequality (4.20) is the best possible in the sense that the constant $\alpha=1 / 3$ cannot be improved in $A^{\alpha} G^{1-\alpha}<L$. This latter point rejoins the fact that if we apply Corollary 4.4 to (4.20) we obtain the same inequality.

Remark 4.3. By virtue of the relationships (4.1), it has been possible to begin by stating and proving the results of the above corollaries and then to deduce those of the corresponding theorems (with discussion on $p$ ). Details of this latter point are omitted for the reader.

Now, we will be interested by bounds of $l_{p}$ in a convex-arithmetic expression as well:

Theorem 4.6. Let $\alpha, \beta \in[0,1]$ be two real numbers such that

$$
\alpha B_{p}+(1-\alpha) G<l_{p}<\beta B_{p}+(1-\beta) G,
$$

for some real number $p$. Then there holds

$$
\begin{gathered}
\alpha\left(\frac{B_{p}^{p}+G^{p}}{2}\right)^{1 / p}+(1-\alpha) G^{1 / 2}\left(\frac{B_{p}^{p}+G^{p}}{2}\right)^{1 / 2 p}<l_{p} \\
<\beta\left(\frac{B_{p}^{p}+G^{p}}{2}\right)^{1 / p}+(1-\beta) G^{1 / 2}\left(\frac{B_{p}^{p}+G^{p}}{2}\right)^{1 / 2 p} .
\end{gathered}
$$

Proof. By the same arguments as previous, we have

$$
\mathcal{R}\left(B_{p}, \alpha B_{p}+(1-\alpha) G, G\right)<l_{p}<\mathcal{R}\left(B_{p}, \beta B_{p}+(1-\beta) G, G\right) .
$$

Again, thanks to Lemma 2.3, we obtain

$$
\begin{gathered}
\alpha\left(B_{p}(\sqrt{a}, \sqrt{b})\right)^{2}+(1-\alpha) G^{1 / 2} B_{p}(\sqrt{a}, \sqrt{b})<l_{p} \\
<\beta\left(B_{p}(\sqrt{a}, \sqrt{b})\right)^{2}+(1-\beta) G^{1 / 2} B_{p}(\sqrt{a}, \sqrt{b}) .
\end{gathered}
$$

By virtue of the identity (4.6), we obtain the desired result after simple manipulations.

As in the above, taking $p=1$ in the latter theorem we immediately obtain the following result.

Corollary 4.7. Let $\alpha, \beta \in[0,1]$ be two real numbers such that

$$
\alpha A+(1-\alpha) G<L<\beta A+(1-\beta) G .
$$

Then there holds

$$
\begin{gathered}
\alpha\left(\frac{A+G}{2}\right)+(1-\alpha)\left(\frac{A G+G^{2}}{2}\right)^{1 / 2}<L \\
\quad<\beta\left(\frac{A+G}{2}\right)+(1-\beta)\left(\frac{A G+G^{2}}{2}\right)^{1 / 2} .
\end{gathered}
$$

Theorem 4.6 has many interesting consequences. For instance, we give the two following corollaries.

Corollary 4.8. Let $\alpha \in[0,1]$ be such that

$$
L<\alpha A+(1-\alpha) G .
$$


Then we have

$$
L<\frac{1+\alpha}{4} A+\frac{3-\alpha}{4} G .
$$

If $\alpha>1=3$ then (4.34) refines (4.33).

Proof. According to Theorem 4.6, we have

$$
L<\alpha\left(\frac{A+G}{2}\right)+(1-\alpha)\left(\frac{A G+G^{2}}{2}\right)^{1 / 2} .
$$

If we write

$$
\left(\frac{A G+G^{2}}{2}\right)^{1 / 2}=G^{1 / 2}\left(\frac{A+G}{2}\right)^{1 / 2}
$$

and we apply the arithmetic-geometric mean inequality, i.e.,

$$
G^{1 / 2}\left(\frac{A+G}{2}\right)^{1 / 2}<\frac{1}{2} G+\frac{1}{2} \frac{A+G}{2},
$$

we obtain the announced result after substituting this latter inequality in (4.35). If $\alpha$ $>1 / 3$, it is easy to see by similar manner as previous that (4.34) refines (4.33) and the proof is completed.

Corollary 4.9. The following inequality holds true

$$
L<\frac{1}{3} A+\frac{2}{3} G
$$

Proof. Similarly to the above, it is sufficient to see that the sequence $\left(\alpha_{n}\right)$ defined by

$$
\alpha_{n+1}=\frac{1+\alpha_{n}}{4}, \quad \text { with } \alpha_{0} \in[0,1]
$$

converges to $1 / 3$ and the desired result follows as previous. We omit the routine detail here.

Remark 4.4. The inequality (4.38) was differently proved by Carlson [12] and here obtained by the same approach as (4.20) and (4.26).

Let us illustrate the above theoretical examples with the following examples.

Example 4.3. Consider the above mean-inequality $L<(1 / 3) A+(2 / 3) G$ which corresponds to $\alpha=1 / 3$ in Corollary 4.8. With this, the obtained refinement is given by

$$
L<\frac{1}{3}\left(\frac{A+G}{2}\right)+\frac{2}{3}\left(\frac{A G+G^{2}}{2}\right)^{1 / 2}<\frac{1}{3} A+\frac{2}{3} G .
$$

Of course, we can combine some the above results to improve the lower and upper bounds of $L$. The following example explains this situation.

Example 4.4. Let us consider the following double inequality

$$
A^{1 / 3} G^{2 / 3}<L<\frac{1}{3} A+\frac{2}{3} G
$$


Combining Theorems 4.1 and 4.6 we immediately obtain

$$
G^{1 / 3}\left(\frac{1}{2} A+\frac{1}{2} G\right)^{2 / 3}<L<\frac{1}{3}\left(\frac{1}{2} A+\frac{1}{2} G\right)+\frac{2}{3}\left(\frac{1}{2} A G+\frac{1}{2} G^{2}\right)^{1 / 2} .
$$

The reader can easily verify that this latter double inequality refines the initial one, so proving our desired aim.

Theorem 4.10. Let $\alpha \in[0,1]$ be such that

$$
l_{p}<\alpha B_{p}+(1-\alpha) G
$$

for some $p \leq 1$. Then there holds

$$
l_{p}<\frac{1+\alpha}{4} B_{p}+\frac{3-\alpha}{4} G .
$$

Proof. If (4.43) holds then Theorem 4.6 gives

$$
l_{p}<\alpha\left(\frac{B_{p}^{p}+G^{p}}{2}\right)^{1 / p}+(1-\alpha) G^{1 / 2}\left(\frac{B_{p}^{p}+G^{p}}{2}\right)^{1 / 2 p}
$$

This, with $p \leq 1$ and the monotonicity of power means, yields

$$
l_{p}<\alpha\left(\frac{B_{p}+G}{2}\right)+(1-\alpha) G^{1 / 2}\left(\frac{B_{p}+G}{2}\right)^{1 / 2} .
$$

The arithmetic-geometric mean inequality gives

$$
G^{1 / 2}\left(\frac{B_{p}+G}{2}\right)^{1 / 2}<\frac{1}{2} G+\frac{1}{2} \frac{B_{p}+G}{2}
$$

and the desired inequality follows by combining (4.46) and (4.47) with a simple reduction.

Taking $p=-1$ in the above theorem, with the fact that $l_{-1}=L^{*}=G^{2} / L$ and $B_{-1}=H$ $=G^{2} / A$, we immediately obtain the next result.

Corollary 4.11. Let $\alpha \in[0,1]$ be such that

$$
\frac{1}{L}<\frac{\alpha}{A}+\frac{1-\alpha}{G} \text {. }
$$

Then one has

$$
\frac{1}{L}<\frac{1+\alpha}{4} \frac{1}{A}+\frac{3-\alpha}{4} \frac{1}{G} .
$$

If moreover $\alpha>1 / 3$ then (4.49) refines (4.48).

Theorem 4.12. For all real number $p \leq 1$ with $p \neq 0$, we have

$$
l_{p}<\frac{1}{3} B_{p}+\frac{2}{3} G
$$

In particular, the following inequality holds

$$
\frac{1}{L}<\frac{1}{3} \frac{1}{A}+\frac{2}{3} \frac{1}{G} \text {. }
$$

Proof. We left it to the reader as an interesting exercise. 
We end this section by stating another result showing how to obtain a lower bound of the logarithmic mean $L$ when we start from an upper bound of its dual $L^{*}$. In fact, since $L^{*}$ is $(A, H)$-stabilizable then we search bounds of $L^{*}$ in terms of $A$ and $H$. Precisely, we have the following.

Theorem 4.13. Let $\alpha$ be a real number satisfying that

$$
L^{*}<\alpha A+(1-\alpha) H .
$$

Then we have

$$
L^{*}<\left(\frac{1+\alpha}{4}\right) A+\left(\frac{3-\alpha}{4}\right) H .
$$

If moreover $\alpha>1 / 3$ then (4.53) refines (4.52).

Proof. Since $L^{*}$ is $(A, H)$-stabilizable then we obtain, with Proposition 2.2,

$$
L^{*}<\mathcal{R}(A, \alpha A+(1-\alpha) H, H)=\alpha \mathcal{R}(A, A, H)+(1-\alpha) \mathcal{R}(A, H, H) .
$$

Thanks to relationships (2.5) for obtaining

$$
L^{*}<\alpha\left(\frac{A+H}{2}\right)+(1-\alpha)\left(\frac{3}{4} A+\frac{1}{4} H\right)^{*} .
$$

Due to the point-wise convexity of the mean-map $m \mapsto m^{*}$, with $A^{*}=H$ and $H^{*}=A$, we obtain

$$
L^{*}<\alpha\left(\frac{A+H}{2}\right)+(1-\alpha)\left(\frac{3}{4} H+\frac{1}{4} A\right),
$$

which after reduction yields the desired result.

Corollary 4.14. The following inequality holds true

$$
\frac{1}{L}<\frac{2}{3} \frac{1}{A}+\frac{1}{3} \frac{1}{H}
$$

Proof. Similarly to the same idea as in the above we have

$$
L^{*}<\left(\frac{1+\alpha_{n}}{4}\right) A+\left(\frac{3-\alpha_{n}}{4}\right) H,
$$

where $\left(\alpha_{n}\right)_{n}$ is the sequence defined by the same recursive relation as in the proof of Corollary 4.9. Letting $n \rightarrow+\infty$ we obtain

$$
L^{*}<\frac{1}{3} A+\frac{2}{3} H
$$

The general relation $m^{*}=G^{2} / m$ valid for all mean $m$, gives in particular, $L^{*}=G^{2} / L$, $H=G^{2} / A$ and $A=G^{2} / H$. Substituting this in the latter inequality, we obtain the desired result.

Remark 4.5. The inequality (4.57) was differently proved by Chen [5] and shown here by the same approach as (4.20), (4.26), and (4.38), so proving the interest of this study. Further, we notice that it is easy to verify that (4.57) is stronger than (4.51).

\section{Refinements for bounding the means $I_{p}$ and $I$}

In this section, we will state some refinements for the power exponential mean $I_{p}$ in a parallel manner to those for $l_{p}$ already presented in the above section. We immediately 
deduce some refinements for the identric mean $I$. The proofs of the results announced here are often similar to that of the above and we omit the routine details to not lengthen this article.

We begin by stating the following lemma which will be needed later.

Lemma 5.1. Let $m_{1}$ and $m_{2}$ be two means such that

$$
m_{1}<I_{p}<m_{2}
$$

for some $p$. Then, for all $a, b>0$, one has

$$
m_{1}\left(a, B_{p}\right) m_{1}\left(B_{p}, b\right)<I_{p}^{2}(a, b)<m_{2}\left(a, B_{p}\right) m_{2}\left(B_{p}, b\right),
$$

where we set $B_{p}:=B_{p}(a, b)$ for the sake of simplicity.

Proof. Since $I_{p}$ is $\left(G, B_{p}\right)$-stabilizable then Theorem 3.2 yields

$$
\mathcal{R}\left(G, m_{1}, B_{p}\right)<I_{p}<\mathcal{R}\left(G, m_{2}, B_{p}\right) .
$$

By computations as previous we easily deduce the desired result.

Starting from a double inequality $m_{1}<I_{p}<m_{2}$, we may choose convenient means $m_{1}$ and $m_{2}$ giving easy computations with the fact that $I_{p}$ is $\left(G, B_{p}\right)$-stabilizable. It is easy to see that $B_{p}<I_{p}<G$ for $p<0$, with reversed inequalities if $p>0$. Then, as for $l_{p}$, bounds of $I_{p}$ in the form $B_{p}^{\alpha} G^{1-\alpha}$ (resp., $\alpha B_{p}+(1-\alpha)$ G) exist for some $\alpha \in[0,1]$. The following result is an analog of Theorem 4.1 from $l_{p}$ to $I_{p}$.

Theorem 5.2. Let $\alpha, \beta\lfloor[0,1]$ be two real numbers such that

$$
B_{p}^{\alpha} G^{1-\alpha}<I_{p}<B_{p}^{\beta} G^{1-\beta},
$$

for some $p$. Then the following double inequality holds

$$
B_{p}^{1-\alpha} G^{1-\alpha}\left(\frac{3}{4} B_{p}^{2 p}+\frac{1}{4} G^{2 p}\right)^{\frac{\alpha}{p}}<I_{p}^{2}<B_{p}^{1-\beta} G^{1-\beta}\left(\frac{3}{4} B_{p}^{2 p}+\frac{1}{4} G^{2 p}\right)^{\frac{\beta}{p}} .
$$

Proof. Since $I_{p}$ is $(G, B p)$-stabilizable then similarly to the above we have

$$
\mathcal{R}\left(G, B_{p}^{\alpha} G^{1-\alpha}, B_{p}\right)<I_{p}<\mathcal{R}\left(G, B_{p}^{\beta} G^{1-\beta}, B_{p}\right) .
$$

Computing as previous and using Lemma 5.1 we obtain

$$
\begin{gathered}
B_{p}^{1-\alpha} G^{1-\alpha}\left(\frac{a^{p}+B_{p}^{p}}{2}\right)^{\alpha / p}\left(\frac{b^{p}+B_{p}^{p}}{2}\right)^{\alpha / p}<I_{p}^{2} \\
<B_{p}^{1-\beta} G^{1-\beta}\left(\frac{a^{p}+B_{p}^{p}}{2}\right)^{\beta / p}\left(\frac{b^{p}+B_{p}^{p}}{2}\right)^{\beta / p} .
\end{gathered}
$$

The desired result follows after a simple reduction, with the fact that

$$
\left(\frac{a^{p}+B_{p}^{p}}{2}\right)\left(\frac{b^{p}+B_{p}^{p}}{2}\right)=\frac{3}{4} B_{p}^{2 p}+\frac{1}{4} G^{2 p},
$$

so completes the proof. $\quad \square$

Taking $p=1$ in the above theorem, with the fact that $B_{1}=A$ and $I_{1}=I$, we deduce the

following result for bounding the identric mean $I$. 
Corollary 5.3. Let $\alpha, \beta$ be two real numbers such that

$$
A^{\alpha} G^{1-\alpha}<I<A^{\beta} G^{1-\beta} .
$$

Then there holds

$$
A^{1-\alpha} G^{1-\alpha}\left(\frac{3}{4} A^{2}+\frac{1}{4} G^{2}\right)^{\alpha}<I^{2}<A^{1-\beta} G^{1-\beta}\left(\frac{3}{4} A^{2}+\frac{1}{4} G^{2}\right)^{\beta} .
$$

Example 5.1. Let $p>0$ be a real number, then we have $I_{p}<B_{p}$ and so the above theorem with $\beta=1$ immediately implies that

$$
I_{p}^{2 p}<\frac{3}{4} B_{p}^{2 p}+\frac{1}{4} G^{2 p} .
$$

In particular, for $p=1$ the double inequality (5.10) is reduced to

$$
A G<I^{2}<\frac{3}{4} A^{2}+\frac{1}{4} G^{2},
$$

which refines the arithmetic-identric-geometric mean inequality $G<I<A$.

Theorem 5.4. Let $\alpha \in[0,1]$ be such that

$$
B_{p}^{\alpha} G^{1-\alpha}<(>) I_{p}
$$

for some $p>(<) 0$, respectively. Then one has

$$
B_{p}^{\frac{2+\alpha}{4}} G^{\frac{2-\alpha}{4}}<(>) I_{p}
$$

If moreover $\alpha<(>) 2 / 3$ then (5.14) refines (5.13).

Proof. Similar to that of the above. We left the detail for the reader as an interesting exercise.

As previously, taking $p=1$ in the above theorem we immediately obtain the following result.

Corollary 5.5. Let $\alpha$ be a real number satisfying that

$$
A^{\alpha} G^{1-\alpha}<I .
$$

Then one has

$$
A^{\frac{2+\alpha}{4}} G^{\frac{2-\alpha}{4}}<I .
$$

If moreover $\alpha<2 / 3$ then (5.16) refines (5.15).

Corollary 5.6. Let $p$ be a real number. If $p>0$ then one has

$$
B_{p}^{2 / 3} G^{1 / 3}<I_{p}
$$

If $p<0$ then the above inequality is reversed. In particular the following inequality holds true

$$
A^{2 / 3} G^{1 / 3}<I .
$$

Proof. We proceed by similar manner as previous. The obtained sequence here is $\left(\alpha_{n}\right)_{n}$ such that 


$$
\alpha_{n+1}=\frac{2+\alpha_{n}}{4}, \quad \text { with } \quad \alpha_{0}=\alpha
$$

which converges to $2 / 3$. We conclude by analogs arguments as previous.

Remark 5.1. The inequality (5.18) has been proved by different methods, see [5] for comparison. We left the reader to state analogs ways about inequality (5.17) as in Remark 4.2.

As the reader can verify it, analog of Theorem 4.6 for $I_{p}$ has length expression and makes appear hard computations.

We left to the reader the routine task for considering other mean-inequalities, involving the standard means, in the aim to obtain more lower and/or upper bounds for a stabilizable mean, eventually with some related refinements. As example, we can state the following.

Theorem 5.7. Let $\alpha \in[0,1]$ be a real number such that

$$
A^{\alpha} G^{1-\alpha}<(>) L_{p}
$$

for some $p \geq(\leq) 0$. Then there holds

$$
A^{1-\alpha} G^{1-\alpha}\left(\frac{3}{4} A^{2}+\frac{1}{4} G^{2}\right)^{\alpha}<(>) L_{p}^{2} .
$$

Theorem 5.8. Let $\alpha, \beta \in[0,1]$ be two real numbers such that

$$
\alpha A+(1-\alpha) G<D_{p}<\beta A+(1-\beta) G
$$

for some $p$. Then we have

$$
\begin{gathered}
\alpha \frac{A+B_{p}}{2}+(1-\alpha)\left(\frac{1}{2} A B_{p}+\frac{1}{2} G B_{p}\right)^{1 / 2}<D_{p} \\
<\beta \frac{A+B_{p}}{2}+(1-\beta)\left(\frac{1}{2} A B_{p}+\frac{1}{2} G B_{p}\right)^{1 / 2} .
\end{gathered}
$$

We omit the proofs of the above results here. Of course, for the proof of Theorem 5.7 we use the fact that $L_{p}$ is $\left(B_{p}, A\right)$-stabilizable while that of Theorem 5.8 uses $D_{p}$ is $\left(A, B_{p}\right)$-stabilizable. Some consequences can also be derived from the above theorems in a similar manner as previous. In particular for $p=0$, Theorem 5.7 coincides with Corollary 5.3 while Theorem 5.8 is reduced to Corollary 4.7. We left all these details to the interested reader.

In summary, the stability and stabilizability concepts are good tool for obtaining a lot of mean-inequalities in a short and nice manner. In particular, some mean-inequalities, already differently proved by many authors in the literature, have been here obtained as consequences via a procedure having a general point of view. This shows the interest of this study derived from the stabilizability concept.

Finally, as the reader can remark it, some other means known in the literature have not been mentioned in the above. As example, the Seiffert's mean $P$ defined by

$$
P(a, b)=\frac{b-a}{4 \operatorname{Arctan} \sqrt{\frac{b}{a}}-\pi}, \quad P(a, a)=a,
$$


has not been considered here. In fact, Raïssouli [1] conjectured that the mean $P$ defined by (5.24) is not stabilizable and this problem remains open. In this direction, we indicate a recent article [11] for further comments about this latter point.

Acknowledgements

The author would like to thank the anonymous referees for their useful comments and suggestions.

Competing interests

The author declares that he has no competing interests.

Received: 27 July 2011 Accepted: 7 March 2012 Published: 7 March 2012

\section{References}

1. Raïssouli, M: Stability and stabilizability for means. Appl Math E-Notes. 11, 159-174 (2011)

2. Qian, W-M, Zheng, N-G: An optimal double inequality for means. J Inequal Appl 2010, 11 (2010). Article ID 578310

3. Alzer, H, Qiu, S-L: Inequalities for means in two variables. Archiv der Mathematik. 80(2):201-215 (2003). doi:10.1007/ s00013-003-0456-2

4. Alzer, H, Janous, W: Solution of problem 8*. Crux Mathematicorum. 13, 173-178 (1987)

5. Chen, Ch-P: On some inequalities for means and the second Gautschi-Kershaw's inequality. RGMIA Suppl 11, 1-9 (2008). Art. 6

6. Leach, EB, Sholander, MC: Extended mean values II. J Math Anal Appl. 92(1):207-223 (1983). doi:10.1016/0022-247X(83) 90280-9

7. Raïssouli, M: United explicit form for a game of monotone and chaotic matrix means. Int Electron J Pure Appl Math. 1(4):475-493 (2010)

8. Sāndor, J: A note on some inequalities for means. Archiv der Mathematik. 56(5):471-473 (1991). doi:10.1007/BF01200091

9. Sāndor, J: Two inequalities for means. Int J Math Math Sci. 18(3):621-623 (1995). doi:10.1155/S0161171295000792

10. Sāndor, J: On certain inequalities for means II. J Math Anal Appl. 199(2):629-635 (1996). doi:10.1006/jmaa.1996.0165

11. Raïssouli, M: Sub-stabilizable and super-stabilizable means. (2012, in press)

12. Carlson, BC: The logarithmic mean. Am Math Monthly. 79, 615-618 (1972). doi:10.2307/2317088

doi:10.1186/1029-242X-2012-55

Cite this article as: Raïssouli: Refinements for mean-inequalities via the stabilizability concept. Journal of Inequalities and Applications 2012 2012:55.

\section{Submit your manuscript to a SpringerOpen ${ }^{\odot}$ journal and benefit from:}

Convenient online submission

- Rigorous peer review

- Immediate publication on acceptance

- Open access: articles freely available online

- High visibility within the field

- Retaining the copyright to your article

Submit your next manuscript at $\gg$ springeropen.com 